\title{
Une cause rare de dysurie chez la femme : le léiomyome de l'urètre et du col vésical
}

\author{
Mohamed Hicham Benazzouz, MD; ${ }^{*}$ Karima Laadam, MD; ${ }^{\dagger}$ Younes Essatara, MD; ${ }^{*}$ Hachem El Sayegh, MD; \\ Ali Iken, MD; Lounis Benslimane, MD; ${ }^{*}$ Ahmed Jahid, MD; ${ }^{\dagger}$ Yassine Nouini, MD*
}

*Service d'Urologie A, Hôpital Ibn Sina, CHU Rabat, Maroc ; 'Service d'Anatomo-pathologie, Hôpital lbn Sina, CHU Rabat, Maroc

Cite as: Can Urol Assoc J 2014;8(11-12):e910-2. http://dx.doi.org/10.5489/cuaj.2024 Published online December 15, 2014.

\section{Résumé}

Le léiomyome de l'urètre est une tumeur bénigne rare constituée de tissus musculaires lisses. Cette tumeur se traduit par une variété de signes cliniques ; le recours à l'histologie est donc nécessaire pour poser le diagnostic. Le traitement de choix est l'exérèse chirurgicale. Aucun cas de dégénérescence maligne n'a été signalé jusqu'à présent, mais une récidive locale est possible en cas d'exérèse incomplète. Les auteurs font état d'un nouveau cas de léiomyome intéressant à la fois le col vésical et l'urètre, et se traduisant par la dysurie chez une patiente ; ils proposent également une revue de la littérature concernant cette pathologie.

\section{Introduction}

Le léiomyome de I'urètre est une tumeur bénigne rare ${ }^{1,2}$. Il s'agit d'une tumeur mésenchymateuse qui se développe aux dépens des tissus musculaires lisses. Depuis le premier cas décrit par Buttner en 1984³, seule une quarantaine de cas ont été recensés dans la littérature mondiale ${ }^{4,5}$. La symptomatologie clinique n'est pas spécifique, et le diagnostic repose sur l'étude anatomopathologique. Le traitement est axé sur l'exérèse chirurgicale ${ }^{6}$. Aucun cas de dégénérescence maligne n'a été signalé jusqu'à présent ${ }^{7-10}$, mais des récidives locales après exérèse incomplète ont été décrites ${ }^{11,12}$. Nous faisons état d'une nouvelle observation de léiomyome intéressant à la fois l'urètre et le col vésical, et discutons la prise en charge de cette pathologie.

\section{Observation}

La patiente, une femme ménopausée de 66 ans sans aucun antécédent pathologique particulier, présentait depuis 7 mois une symptomatologie obstructive du bas appareil uri- naire caractérisée par une dysurie d'aggravation progressive, compliquée par un épisode unique d'hématurie caillotante. L'examen clinique a révélé la présence d'une masse charnue de couleur rougeâtre au niveau du méat urétral (fig. 1).

L'urotomodensitométrie (fig. 2) réalisée chez cette patiente a mis au jour un épaississement tissulaire régulier qui semblait prendre naissance au niveau du plancher vésical, faisait saillie dans la lumière vésicale et s'étendait jusqu'à I'urètre. Ce processus couvrait une superficie de $49 \times 31 \mathrm{~mm}$.

À la cystoscopie, on a retrouvé une masse solide, non papillaire, de couleur gris clair aux dépens de la face supérieure du col vésical et de l'urètre. Cette masse, qui rappelait un lobe médian prostatique, faisait saillie dans la lumière urétrale et l'obstruait complètement. Des signes de lutte de type "vessie à cellules et à colonnes » ont également été observés dans la vessie. Nous avons procédé à une résection transurétrale complète de la masse. L'analyse anatomopathologique (fig. 3 et fig. 4) des 25 grammes de tissus réséqués a révélé une prolifération tumorale bénigne composée de cellules musculaires lisses disposées en faisceaux enchevêtrés. L'analyse histologique a conclu à un léiomyome de I'urètre. Un mois après l'intervention, la patiente ne présentait plus de signes obstructifs du bas appareil urinaire, et I'examen clinique était normal.

\section{Discussion}

Le léiomyome de l'urètre a été décrit pour la première fois par Buttner en 1984². Depuis, seule une quarantaine de cas ont été recensés dans la littérature mondiale $\mathrm{e}^{4,5}$. II s'agit d'une tumeur bénigne rare, constituée de tissus musculaires lisses, qui touche les femmes plus que les hommes ${ }^{13}$. Cette tumeur peut survenir à n'importe quel âge, mais elle apparaît le plus souvent entre 36 et 46 ans. L'âge moyen de survenue est de $41 \mathrm{ans}^{7,14}$. La partie proximale de l'urètre est le siège habituel de cette tumeur, mais la partie distale peut également être touchée ${ }^{15}$. 


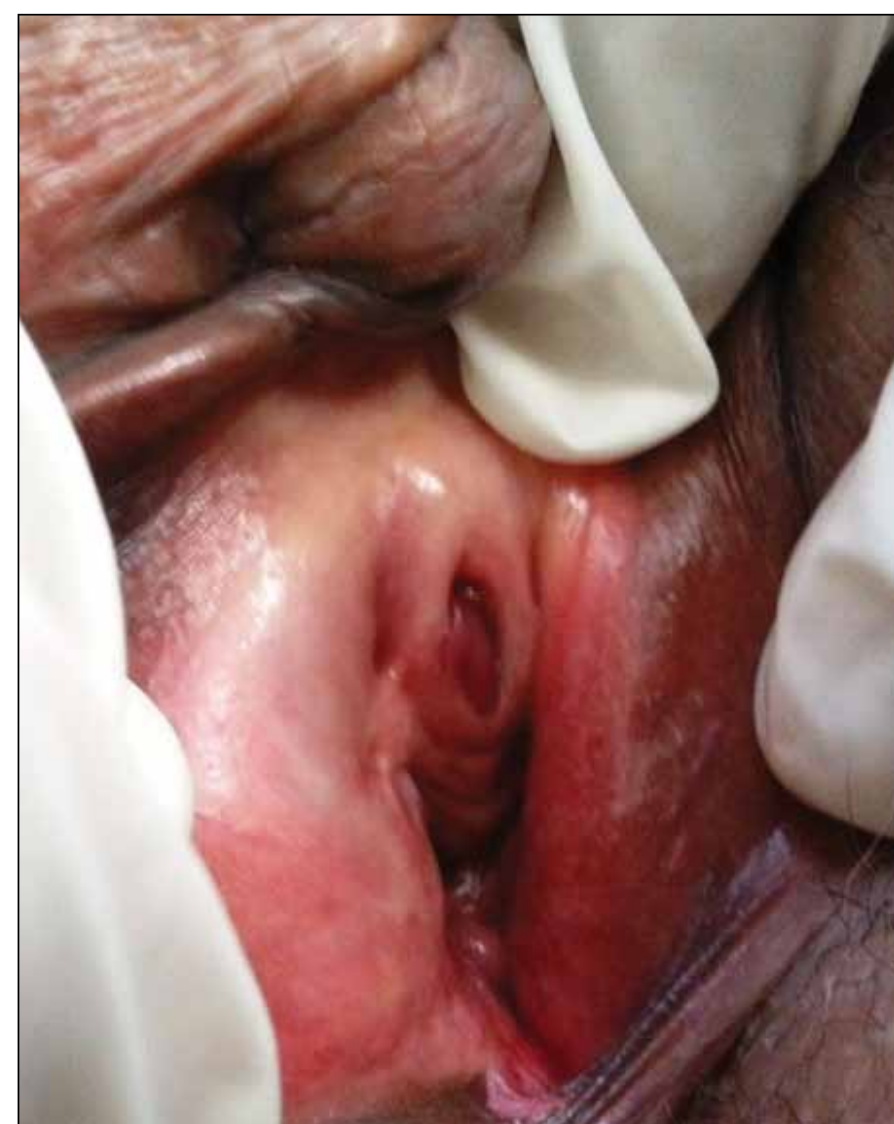

Fig. 1. Présentation clinique du léiomyome de l'urètre : masse charnue, de couleur rougeâtre, au niveau du méat urétral.

L'étiopathogénie des léiomyomes de l'urètre, comme celle des léiomyomes de la vessie, n'est pas encore bien établie. Cependant, quatre hypothèses ont été avancées ${ }^{16}$ :

1) Développement tumoral en rapport avec des perturbations hormonales ;

2) Persistance d'un tissu embryonnaire résiduel dans I'urètre ou la vessie, qui se transforme en léiomyomes ;

3) Transformation métaplasique du réseau vasculaire urétral ou vésical secondaire à une inflammation périvasculaire ;

4) Infection de la musculeuse entraînant une inflammation avec développement de tumeurs bénignes.

La présentation clinique du léiomyome urétral n'est pas spécifique. Les symptômes les plus courants sont les suivants : infections urinaires; effet de masse sur la paroi vaginale antérieure ; dyspareunie ; rétention d'urine ; plus rarement, signes irritatifs du bas appareil urinaire et hématurie macroscopique ${ }^{9,10,17-19}$. Il est à préciser que, dans de nombreux cas, les léiomyomes de l'urètre peuvent rester longtemps asymptomatiques.

Le diagnostic différentiel du léiomyome urétral se fonde sur la présence d'urétrocèles, de caroncules, de diverticules de l'urètre et de tumeurs malignes de l'urètre - d'où l'intérêt de procéder à un examen clinique minutieux, complété

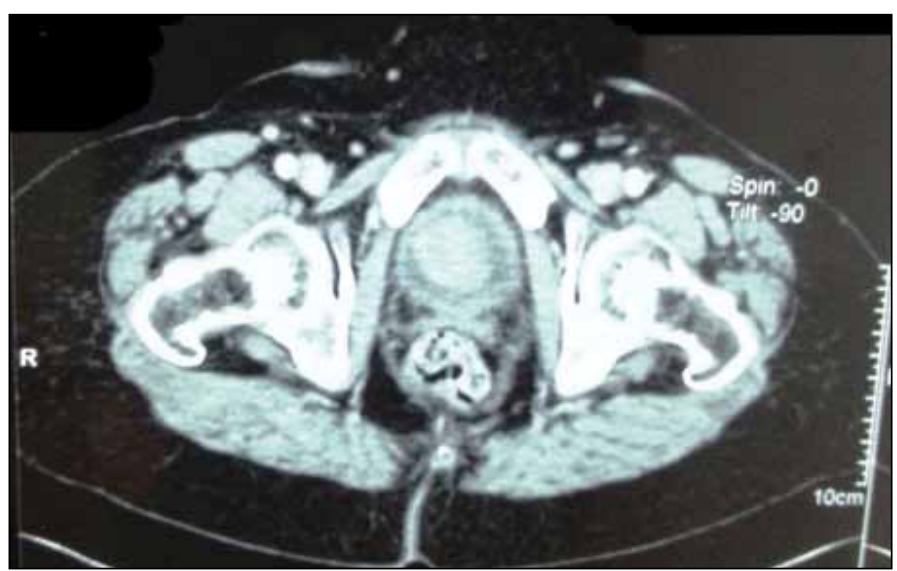

Fig. 2. Coupe tomodensitométrique montrant une masse intravésicale hyperdense près du col vésical.

par des examens d'imagerie et par une urétrocystoscopie. Celle-ci est l'examen clé du diagnostic, car elle permet à la fois de visualiser la tumeur et d'en faire des biopsies. L'urétrocystoscopie doit être minutieuse et inspecter toute la surface urétrale, en particulier chez la femme, dont l'urètre est court et chez qui I'on peut facilement méconnaître une masse urétrale en introduisant directement le fibroscope dans la vessie sans prendre soin de bien examiner l'urètre.

L'imagerie du léiomyome de l'urètre permet de préciser la taille de la tumeur, ses rapports avec les structures avoisinantes et le retentissement sur le haut appareil urinaire. Récemment, l'échographie endovaginale et l'IRM ont également été utilisées pour déterminer la nature bénigne de la tumeur ${ }^{20}$. Cependant, les examens d'imagerie ne peuvent remplacer l'urétrocystoscopie avec biopsie, qui reste la seule intervention permettant de poser un diagnostic de certitude.

À l'urétrocystoscopie, le léiomyome se présente le plus souvent sous la forme d'une masse bien délimitée de couleur gris clair, sessile pour les tumeurs de petite taille et pédiculée pour les tumeurs de plus grande taille ${ }^{21}$. L'analyse histologique révèle des fibres musculaires lisses réparties de manière uniforme, avec moins de neuf mitoses par champ. La présence de plus de neuf mitoses par champ oriente le diagnostic en faveur d'une tumeur maligne ${ }^{21,22}$.

Les traitements de choix sont la résection transurétrale et l'énucléation chirurgicale 9,10,17-19. De rares cas de récidive locale après résection transurétrale ou exérèse incomplète de la tumeur ont été recensés ${ }^{11,12}$, mais aucun cas de dégénérescence maligne $\mathrm{n}^{\prime} \mathrm{a}$ été signalé ${ }^{7-10}$. Certains auteurs proposent une simple surveillance par IRM pour les formes asymptomatiques ${ }^{11}$. L'inconvénient de cette approche est l'absence d'analyse histologique.

\section{Conclusion}

Le léiomyome de l'urètre est une tumeur bénigne rare qui touche les femmes plus que les hommes. L'absence 


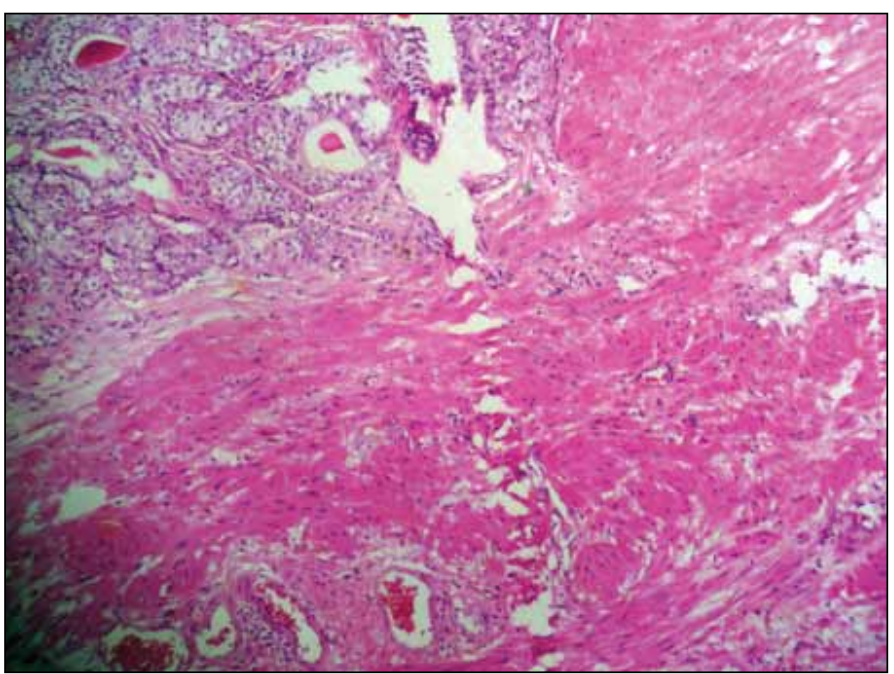

Fig. 3. Paroi urétrale siège d'une prolifération de cellules fusiformes, avec différenciation des cellules musculaires lisses disposées en faisceaux entrecroisés (HE $\times 100)$.

de symptomatologie spécifique rend le diagnostic clinique difficile, si bien que le recours à l'analyse histologique est nécessaire pour poser un diagnostic définitif. L'imagerie sert essentiellement à préciser la taille de la tumeur et ses rapports avec les structures avoisinantes. Le traitement des formes symptomatiques est essentiellement chirurgical, qu'il soit effectué par énucléation ou par résection endoscopique. Aucun cas de dégénérescence maligne n'a été signalé, mais une récidive locale après exérèse incomplète reste possible. Une meilleure connaissance de l'étiopathogénie de cette maladie pourrait offrir aux patients de nouvelles perspectives thérapeutiques locales ou générales visant spécifiquement les cellules de cette tumeur.

Competing interests: Authors declare no competing financial or personal interests.

This paper has been peer-reviewed.

\section{Références}

1. Bai SW, Jung HJ, Jeon MJ, et al. Leiomyomas of the female urethra and bladder: A report of five cases and review of the literature. Int Urogynecol J Pelvic Floor Dysfunct 2007;18:913-7. http://dx.doi. org/10.1007/s00192-006-0257-9

2. Gallego Vilar D, José Povo Martin I, Miralles Aguado J, et al. Leiomyoma of the female urethra, a case and review of the literature. ActasUrol Esp 2010;34:396-7. http://dx.doi.org/10.1016/i. acuro.2010.02.020

3. Blaivas IG, Flisser AJ, Bleustein CB, et al. Periurethral masses: Etiology and diagnosis in a large series of women. Obstet Gynecol 2004;103:842-9. http://dx.doi.org/10.1097/01.A0G.0000124848.63750.e6

4. Bergman A, Karram MM, Bhatia NN, et al. Leiomyoma of the female urethra. A case report. J Reprod Med 1989;34:1001.

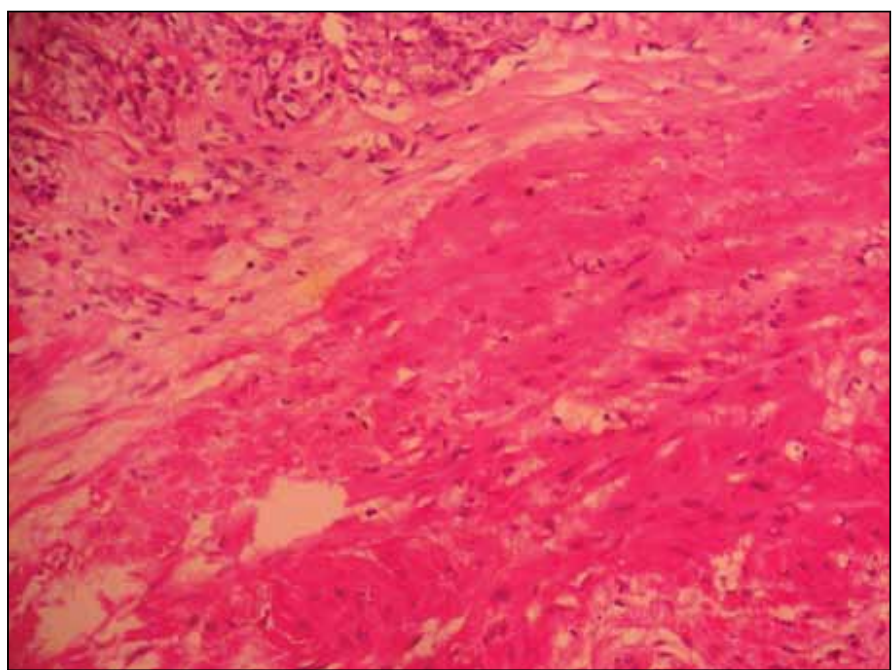

Fig. 4. Prolifération tumorale constituée de cellules musculaires lisses sans atypies cytonucléaires (HE ×200).

5. Dasan JCR, Rao K, Nalini V, et al. Leiomyoma of the female urethra-a clinical curiosity. Int J Gynaec Obst 1989;28:381. http://dx.doi.org/10.1016/0020-7292(89)90613-9

6. Deka PM, Rajeev TP. Leiomyoma of the female urethra: A case report. Urologia Int 2003;71:224-5. http://dx.doi.org/10.1159/000071854

7. Chong KM, Chuang J, Tsai YL, et al. A rapidly growing paraurethral myoma with profuse bleeding from a mucosal vessel: Report of a case. Gynecol Obstet Invest 2006;61;87-9. http://dx.doi. org $/ 10.1159 / 000089010$

8. Jalde DD, Godhi SA, Uppin SM, et al. A case of urethral leiomyoma- a rare presentation. World I Sci Technol 2012;2:27-8.

9. Perera ND, Senanayake L, Vithana $\mathrm{VH}$, et al. An unusual presentation of female urethral leiomyoma. Ceylon Med J 2005;50:31-3.

10. Saad AG, Kaouk JH, Kaspar HG, et al. Leiomyoma of the urethra: Report of 3 cases of a rare entity. Int J Surg Pathol 2003;11:123-6. http://dx.doi.org/10.1177/106689690301100213

11. Fry M, Wheeler JS, Mata JS Jr, et al. Leiomyoma of the female urethra. J Urol 1988; 140:613-7.

12. Lake MH, Kossow AS, Bokinsky G. Leiomyoma of the bladder and urethra. J Urol 1981;125:742.

13. Shield DE, Weiss RM. Leiomyoma of the female urethra. J Urol 1973;109:430-1.

14. Ozel B, Ballard C. Urethral and paraurethral leiomyomas in the female patient. Int Urogynecol J Pelvic Floor Dysfunct 2006;17:93-5. http://dx.doi.org/10.1007/s00192-005-1316-3

15. Lee MC, Lee SD, Kuo HT, et al. Obstructive leiomyoma of the female urethra: Report of a case. J Urol 1995;153:420-1. http://dx.doi.org/10.1097/00005392-199502000-00041

16. Teran AZ, Gambrell RD. Leiomyoma of the bladder. Int J Fertil 1989;34:289-92.

17. Pahwa $M$, Saifee $Y$, Pahwa AR, et al. Leiomyoma of the female urethra-a rare tumor: Case report and review of the literature. Case Rep Urol 2012;2012:280816.

18. Riviere P, Bodin R, Bernard G, et al. Leiomyoma of the female urethra. Prog Urol 2004;14:1196-8.

19. Deka PM, Rajeev TP. Leiomyoma of the female urethra. A case report. Urol Int 2003;71:224-5. http:// dx.doi.org/10.1159/000071854

20. Pavlica $\mathrm{P}$, Bartolone A, Gaudiano C, et al. Female paraurethral leiomyoma: Ultrasonographic and magnetic resonance imaging findings. Acta Radiologica 2004;45:796-8. http://dx.doi. org/10.1080/02841850410001376

21. Chang SD, Cooperberg PL, Wong AD, et al. Limited-sequence magnetic resonance imaging in the evaluation of the ultrasonographically indeterminate pelvic mass. Can Assoc Radiol J 2004;55:87-95.

22. Joshi HB, Beck RO. Leiomyoma of the female urethra with upper tract dilatation and treatment with transurethral resection: A case report and literature review. Tech Urol 2000;6:223-8.

Correspondence: Dr. Mohamed Hicham Benazzouz, Service d'Urologie A, Hôpital lbn Sina, CHU Rabat, Maroc; benazzouzhicham@gmail.com 